\title{
Modelling of Non-coaxial Viscoplastic Deformation in
}

\section{Geodynamics}

\author{
Hans B. Muhlhaus ${ }^{1}$, Louise Olsen-Kettle ${ }^{1}$, Jingyu $\mathrm{Shi}^{1}$ and Louis Moresi ${ }^{2,3}$ \\ 1. School of Earth Sciences, The University of Queensland, Brisbane 4072, Australia \\ 2. School of Mathematical Sciences, Monash University, Clayton 3800, Australia \\ 3. School of Geosciences, Monash University, Clayton 3800, Australia
}

\begin{abstract}
The formation of shear bands for time and length scales appropriate for deformation processes in the upper Lithosphere is investigated in plane strain finite element simulations under predominantly uniaxial extension and compression, respectively. The direction of gravity is assumed orthogonal to the extension/compression axis. Mathematically, the formation of shear zones may be explained as a consequence of changes in the type of the governing model equations. Such changes or bifurcations depend strongly on the details of the constitutive relationships such as strain softening, thermal or chemical effects, associated or non-associated - coaxial or non-coaxial flow rules. Here we focus on strain softening and coaxial and non-coaxial flow rules. In the simulations, we consider an initially rectangular domain with the dimensions $L_{0}, H_{0}$ in the horizontal, vertical directions, respectively. The domain is extended or compressed by prescribing a uniform, horizontal velocity field along one of the vertical boundaries while keeping the opposite boundary fixed. An important global descriptor of the deformation process is the relationship between the horizontal stress resultant (average horizontal stress) and the strain $\ln \left(L / L_{0}\right)$, where $L$ is the deformed length of the domain. The main goal of this paper is to investigate key factors influencing the phenomenology of the localization process such as flow rule, coaxial, non-coaxial and strain softening. Different origins of the mesh sensitivity of deformations involving localization are also investigated.
\end{abstract}

Key words: Shearing band, non-coaxiality, softening, viscous plastic materials.

\section{Introduction}

On a global scale, the continents drift as an integral part of the surface thermal boundary layer of the convection mantle. They have retained a distinct identity within the mantle flow for billions of years while developing a strong physical and chemical fabric along the way. Motions in the mantle are described by the equations of fluid dynamics for very large deformation. The rheology needed to describe deformation in the lithosphere is highly non-linear, and near the surface, where temperatures are less than approximately $600{ }^{\circ} \mathrm{C}$, it becomes necessary to consider the role of plasticity [1, 2] and elasticity [3]. Structures experiencing large elastic-plastic deformations typically undergo a succession of

Corresponding author: Hans Muhlhaus, professor, research fields: mechanics, computational mechanics and geomechanics. E-mail: h.muhlhaus@uq.edu.au. instabilities. For instance, a plate in extension will deform initially in a uniform fashion until, at a critical stress level, a neck forms around the symmetry axis of the deformation [4-11]. Subsequently, shear bands form within the region of the neck. Once the shear bands are established, the deformation of the plate is carried almost exclusively by the deformations of the bands, more or less passively accommodated by the elastic deformations of the surrounding material.

Shear bands or faults are arguably the most important deformation mechanism in the upper lithosphere. They occur on many different scales, e.g., in the form of detachment faults in rift zones or as collapse mechanisms of geotechnical structures [12]. The stress and temperature conditions under which shear bands form depend strongly on the local material behavior and as such on the details of the constitutive relationships. If the flow rule is non-associated, e.g., if the pressure 
sensitivity is not equal to the dilatancy factor relating the inelastic volume change to the equivalent plastic strain rate, then shear banding is possible under increasing stress, in the hardening regime $[13,14]$. The stress level and the preferred band orientation also depend on whether the plastic strain rate has the same principal axes as the stress. If the latter is the case then the flow rule is designated as co-axial. Non-coaxial models have been proposed originally by de Josselin de Jong [15, 16] and Spencer $[17,18]$ in the context of granular materials. For Spencer the main motivation to consider non-coaxiality seemed to be the desire for a theory in which the stress and the velocity characteristics coincide. A number of researchers have considered the theory, looking at new derivations, various motivations and considering extensions [19-21]. A recent review is shown by Tordesillas et al. [22].

In this paper, we will explore the role of non-coaxiality on shear banding in pure shear in compression and extension. The deformation and localization process is illustrated by results of large deformation finite element simulations for different sets of the strength parameters and loading rates. To facilitate quantitative comparisons, we also calculate the relationship between the average horizontal stress (stress resultant) conjugate to the prescribed boundary velocity and a strain measure for the horizontal extension of the (initially) rectangular domain under consideration. We assume incompressible deformations since the emphasis in this study is on large deformations. The equations of motion are integrated using an updated Lagrangian scheme. For the co-axial case we compare our results with previous results by Lemiale et al. [23].

\section{Constitutive Relationships}

\subsection{Rigid Plastic Model}

We derive a simple basic plane-strain non-coaxial rigid plastic model by assuming that the inelastic deformation is carried by a single slip system (Fig. 1), which is oriented under either $(\pi / 4+v / 2)$ or $-(\pi / 4+$ $v / 2$ ) to the direction of the largest (least compressive) principal stress. The angle $v$ is the angle of non-coaxiality $[15,17,24]$, which is the angle between the direction of largest principal stress and the direction of largest principal strain rate. The flow rule is coaxial if $v=0$ and non-coaxial if $v>0$. The flow rules proposed by Harris [25] and Moresi and Muhlhaus [9] are obtained for $v=\phi$, where $\phi$ is the Mohr-Coulomb angle of friction. The following relationship exists between the plastic stretching $D_{i j}^{p}$, the shear and normal vectors $\left(s_{i}\right.$ and $n_{j}$, respectively) in the drawing plane, and the shear strain rate $\dot{\gamma}^{p}$ :

$$
D_{i j}^{p}=\frac{\dot{\gamma}^{p}}{2}\left(s_{i} n_{j}+s_{j} n_{i}\right)
$$

The components of the shear and the normal vector in Eq. (1) depend on the orientation of the shear mechanism relative to the principal stress axes. In Fig. 1, the shear and normal vectors corresponding to the orientations $+/-(\pi / 4+v / 2)$ are marked by the indexes $(a)$ and $(b)$, respectively. The components of the shear and normal vector can be expressed in terms of trigonometric functions of $\varphi \pm(\pi / 4+v / 2)$ where, $\varphi$ is the angle between the spatial coordinate system and the axis of the larger principal stress (Fig. 1). Using the well-known relationships for the principal stress angle $\varphi$ :

$$
\begin{aligned}
& \tan 2 \varphi=\frac{2 \sigma_{12}}{\sigma_{11}-\sigma_{22}} \\
& \sin 2 \varphi=\frac{\sigma_{12}}{\tau} \\
& \cos 2 \varphi=\frac{\sigma_{11}-\sigma_{22}}{2 \tau}
\end{aligned}
$$

where, $\tau=\sqrt{\left(\sigma_{11}-\sigma_{22}\right)^{2}+4 \sigma_{12}^{2}} / 2$, we obtain for the plastic stretching:

$$
D^{p}=\frac{\dot{\gamma}^{p}}{2 \tau}\left(\cos v\left[\begin{array}{cc}
\left(\sigma_{11}-\sigma_{22}\right) / 2 & \sigma_{12} \\
\operatorname{symm} & -\left(\sigma_{11}-\sigma_{22}\right) / 2
\end{array}\right] \pm \sin v\left[\begin{array}{cc}
-\sigma_{12} & \left(\sigma_{11}-\sigma_{22}\right) / 2 \\
\operatorname{symm} & \sigma_{12}
\end{array}\right]\right)
$$




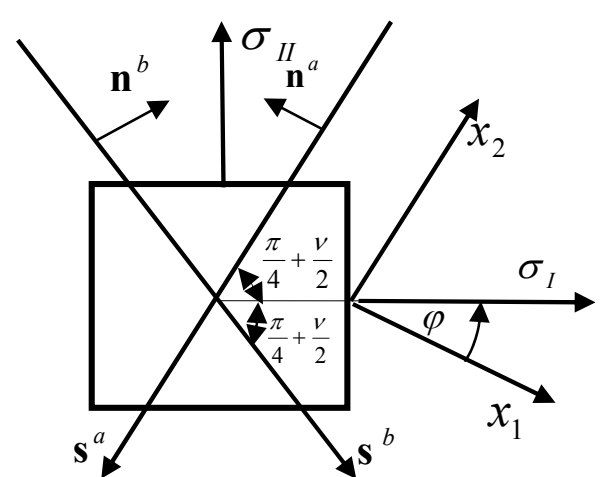

Fig. 1 Principal stresses $\left(\sigma_{I}, \sigma_{I I}\right)$ and slide systems $\left(s^{a}, n^{a}\right)$ and $\left(s^{b}, n^{b}\right) ;\left(x_{1}, x_{2}\right)$ are the coordinates of a global, spatially fixed Cartesian coordinate system.

where the + sign applies if $(\pi / 4+v / 2)$ is active, and the - sign applies if $-(\pi / 4+v / 2)$ is active. Alternatively Eq. (3) may be written as:

$$
D_{i j}^{p}=\frac{\dot{\gamma}^{p}}{2 \tau}\left(\cos v \sigma_{i j}^{\prime} \pm \sin v \sigma_{i j}^{\perp}\right)
$$

where, $\sigma_{i j}=\sigma_{i j}^{\prime}+p \delta_{i j}$ with $p=-\left(\sigma_{11}+\sigma_{22}\right) / 2$ and $\delta_{i j}$ being the Kronecker delta tensor, and $\sigma_{i j}^{\perp}=Q_{\theta=\pi / 4} \sigma^{\prime} Q_{\theta=\pi / 4}^{T}$,

with

$$
Q=\left[\begin{array}{cc}
\cos \theta & -\sin \theta \\
\sin \theta & \cos \theta
\end{array}\right]
$$

It can be shown that $\sigma_{i j}^{\prime} \sigma_{i j}^{\perp}=0$ and

$$
\tau=\sqrt{\sigma_{i j}^{\prime} \sigma_{i j}^{\prime} / 2}=\sqrt{\sigma_{i j}^{\prime \perp} \sigma_{i j}^{\prime \perp} / 2}
$$

From Eqs. (4) and (6), it follows that:

$$
\dot{\gamma}^{p}=\sqrt{2 D_{i j}^{p} D_{i j}^{p}}
$$

We assume that plastic flow takes place if the Mohr-Coulomb yield criterion is satisfied. In this case we have:

$$
\dot{\gamma}^{p}=\left\{\begin{array}{l}
>0 \text { if } \tau=c \cos \phi+p \sin \phi \\
=0 \text { if } \tau<c \cos \phi+p \sin \phi
\end{array}\right.
$$

where, $c$ is the cohesion and $\phi$ is the angle of friction.

\subsection{Viscous-Plastic Model for Single Slip}

We follow the standard approach by decomposing the total strain rate into a viscous and a plastic part, i.e.,

$$
D_{i j}=D_{i j}^{v}+D_{i j}^{p}
$$

We define $D_{i j}^{p}$ according to Eq. (4) and for the viscous part of the stretching we assume $D_{i j}^{v}=\sigma_{i j}^{\prime} / 2 \eta$; insertion into Eq. (9) yields:

$$
D=\frac{1}{2}\left(\frac{1}{\eta \cos v}+\frac{1}{\eta^{p}}\right) \cos v \sigma_{i j}^{\prime} \pm \frac{1}{2 \eta^{p}} \sin v \sigma_{i j}^{\perp}
$$

The plastic viscosity is defined as following: $\eta^{p}=(c \cos \phi+p \sin \phi) / \dot{\gamma}^{p}$.

Inverting Eq. (10) for the deviatoric stress $\sigma_{i j}^{\prime}$ yields:

$$
\begin{aligned}
\sigma_{i j}^{\prime}= & \frac{2 \eta^{p}\left(\eta^{p} / \eta+\cos v\right)}{(\sin v)^{2}+\left(\eta^{p} / \eta+\cos v\right)^{2}} \\
& \times\left(D_{i j} \mp \frac{\sin v}{\eta^{p} / \eta+\cos v} D_{i j}^{\perp}\right)
\end{aligned}
$$

The tensor $D_{i j}^{\perp}$ is defined in analogy to $\sigma_{i j}^{\perp}$ as $D^{\perp}=Q_{\pi / 4} D Q_{\pi / 4}^{T}$.

For $v=0$, Eq. (11) reduces to a standard, co-axial, viscous-plastic model. In the rigid-plastic limit, $\eta \rightarrow \infty$, we recover the rigid plastic model (4).

Eq. (11) may be rewritten in a form more amenable for numerical analysis as:

$$
\sigma_{i j}^{\prime}=L_{i j k l} D_{k l}
$$

with

$$
L_{i j k l}=\frac{\eta^{p}\left(\eta^{p} / \eta+\cos v\right)}{(\sin v)^{2}+\left(\eta^{p} / \eta+\cos v\right)^{2}}\left(\delta_{i k} \delta_{j l}+\delta_{i l} \delta_{j k} \mp \frac{\sin v}{\eta^{p} / \eta+\cos v}\left(Q_{i k} Q_{j l}+Q_{i l} Q_{j k}\right)\right)
$$

For the rotation tensor $Q$ in Eq. (13), we assume a rotation of $\pi / 4$ as in the first relationship of Eq. (5); the subscript $\pi / 4$ has been dropped for convenience of notation. The tensor $L$ is symmetric with respect to interchange of $I \leftrightarrow j, k \leftrightarrow j$ as it should, however, $L$ is not invariant with respect to an interchange of the indexes $i \leftrightarrow k, j \leftrightarrow l$. This, however, means that the corresponding boundary value problems are non self-adjoint, i.e., the stiffness matrix in finite element calculations is non-symmetric which is similar to the 
situation in non-associated plasticity. Indeed, the plastic flow rule given as Eq. (3) or Eq. (4) is non-associated.

Recently, Muhlhaus et al. [26] formulated a non-coaxial model based on double slip mechanism within the framework of a Cosserat continuum theory. For the double slip model, there are two plastic strain rates governing the flow rule, only one of which can be determined from the yield criterion. A closure for the other is proposed.

\subsection{Softening Model}

The effect of material damage accumulation, e.g., through micro-crack growth or porosity nucleation is considered by the following simple strain softening relationship proposed by Moresi and Muhlhaus [9]:

$$
c=c_{0}+\left(c_{\infty}-c_{0}\right) \min \left(1, \gamma^{p} / \gamma_{\infty}\right)
$$

where, $c_{0}$ is the initial cohesion, $c_{\infty}$ is the residual cohesion and $\gamma^{p}$ is the accumulated plastic strain, i.e., $d \gamma^{p} / d t=\sqrt{2 D_{i j}^{p} D_{i j}^{p}}$; the parameter $\gamma_{\infty}$ is the value of the accumulated plastic strain at which the cohesion assumes its residual value.

\section{Extension and Compression of a Rectangular Layer}

We conduct numerical simulations on a rectangular domain with initial dimensions $\left(L_{0}, H_{0}\right)$ under extension or compression in horizontal $x_{1}$-direction. The simulation is carried with non-dimensionalized governing equations, with the density of the material $\rho$ $=1$, and all parameters are non-dimensional. Gravity is an external force in vertical $x_{2}$-direction acting in the domain. All boundaries are assumed as smooth, i.e., no shear stresses. The top boundary is assumed as stress free. The vertical velocity is assumed to be zero at the bottom boundary. The velocities in $x_{1}$-direction $V_{1}=V$ and $V_{1}=-V$ are prescribed at the right and left boundaries, respectively, where $V>0$ in the extension case and $V<0$ in the compression case. The initial dimensions of the domain are precribed as $\left(L_{0}, H_{0}\right)=(3$,
$1)$ in extension and $(4,1)$ in compression, respectively. We also assume a small weak zone $(0.04 \times 0.04)$ with weaker constant viscosity $\eta_{w}=\eta / 100$ located at the middle of the bottom boundary, where, $\eta=100$ is the viscosity of the surrounding material. We choose the internal friction $\tan \phi=0.4(0.6)$, the initial cohesion $c_{0}$ $=4.0(20.0)$, the residual cohesion $c_{\infty}=2.0$ (10.0) for extension (compression) cases respectively, and $\gamma_{\infty}=$ 0.1 . The boundary velocity is $V=0.035(-0.5)$ for extension (compression).

\subsection{Bounding Solutions for Stress Resultants}

In this section, we derive static bounding solutions for the extension and compression cases considered below. In the extension case, we have $\sigma_{11}>0>\sigma_{22}$ and from the yield criterion $\tau-p \sin \phi-c \cos \phi \leq 0$ it follows for plastic limit states:

$$
\sigma_{11}=\frac{1-\sin \phi}{1+\sin \phi} \sigma_{22}+\frac{2 c \cos \phi}{1+\sin \phi}
$$

Under gravity, we have:

$$
\sigma_{22}=-\rho g\left(H-x_{2}\right)
$$

and, for the stress resultant $\sigma=1 / H \int_{0}^{H} \sigma_{11} d x_{2}$, we obtain the expression:

$$
\sigma=-\frac{\rho g H}{2} \frac{1-\sin \phi}{1+\sin \phi}+\frac{2 c \cos \phi}{1+\sin \phi}
$$

It can be seen that from Eq. (17), in the extension case, the stress resultant is negative (i.e., compressive).

$$
\rho g H>\frac{4 c \cos \phi}{1-\sin \phi}
$$

Under extensive conditions, the stress becomes compressive once the cohesion is sufficiently small according to the inequality Eq. (18).

For the compressive case in the plastic limit state, $\sigma_{11}<\sigma_{22}<0$, we obtain:

$$
\sigma=-\left(\frac{\rho g H}{2} \frac{1+\sin \phi}{1-\sin \phi}+2 c \frac{\cos \phi}{1-\sin \phi}\right)
$$

\subsection{Numerical Simulation and Velocity Calibration}

In the simulations, we use quadrilateral 4-noded 
element with selective integration: 4 gauss points for the velocity degrees of freedom and one (central) Gauss point for the pressure. This simple, so called selective integration method is known to produce adequate answers to incompressible deformation problems although the method does not pass the B-B (Brezzi-Babushka) condition [27]. The pressure corresponding to the incompressible deformation is solved with penalty scheme. The angle of non-coaxiality is assumed to be constant in the numerical simulation. In addition to the yield criterion: $\tau-p \sin \phi-c \cos \phi=0$, we also employ pressure cutoff at $p=-c \cos \phi / \sin \phi$. The domain is discretised into $384 \times 128$ elements.

We will derive an estimate for the prescribed boundary velocity in the extension case which would guarantee plastic deformations in the absence of the imperfection at $\left(L_{0} / 2,0\right)$. From the constitutive relation in the viscous regime, we have $\sigma_{11}=\sigma_{22}+4 \eta D_{11}=\sigma_{22}+8 \eta V / L$. At the onset of plastic deformation this stress should be equal to that given by Eq. (15). To guarantee plastic deformation the velocity $V$ should ensure that this stress is not less than the latter. With $\sigma_{22}$ given by Eq. (16) and integrating these expressions with respect to $x_{2}$ in the range $0 \leq x_{2} \leq H$, we obtain:

$$
V \geq \frac{L}{8 \eta}\left(\rho g H \frac{\sin \phi}{1+\sin \phi}+\frac{2 c \cos \phi}{1+\sin \phi}\right)
$$

Using the parameters given above the condition in Eq. (20) is satisfied for $V=0.03$ and in the simulations we use $V=0.035$.

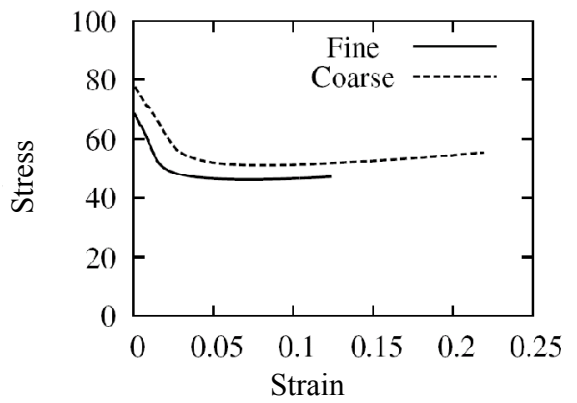

(a)

\subsection{Mesh Sensitivity}

Because of strain softening, mesh sensitivity of the numerical solution is present. The reason for this kind of mesh sensitivity which is only indirectly related to the usual discretisation error has been well described by Schreyer [28]. The critical property of the softening related mesh sensitivity, namely zero plastic dissipation upon mesh refinement can be removed by including the effect of elasticity [29] or by enriching the physics of the mechanical model by inclusion of additional, micromechanical degrees of freedom [30]. The present simple viscous-plastic model seems adequate however in the context of large plastic deformations where the initial softening part of the deformation process represents only a small part of the total deformation.

The relationship between the average horizontal stress $\sigma$ and the average horizontal strain $\varepsilon=\ln \left(L / L_{0}\right)$ is represented for two levels of mesh refinement in Fig. 2. The finer mesh has $384 \times 128$ elements and the coarse mesh has $192 \times 64$ elements. Fig. 2a shows the variation of the stress with strain for compression with non-coaxial rule and softening, while Fig. $2 \mathrm{~b}$ shows the variation for extension with non-coaxial rule and softening. It can be seen that it is sensitive to the mesh size.

\section{Effects of Non-coaxiality and Softening}

\subsection{Effects in Compression}

We first consider simulation of the compression of the rectangular layer. Fig. 3a shows the variation of the

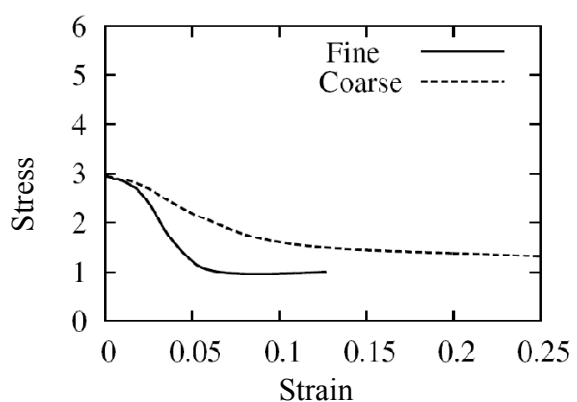

(b)

Fig. 2 Variation of average normal stress with strain for two meshes under (a) compression and (b) extension. 


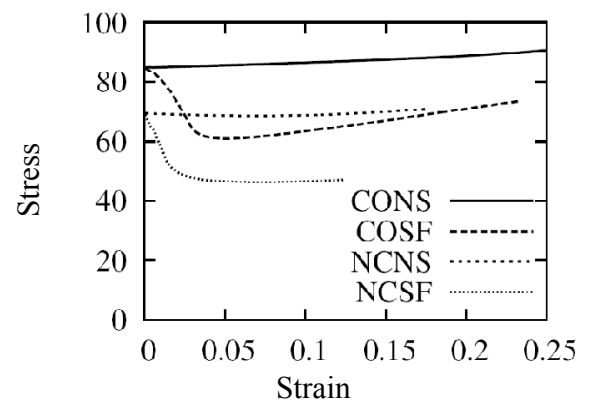

(a)

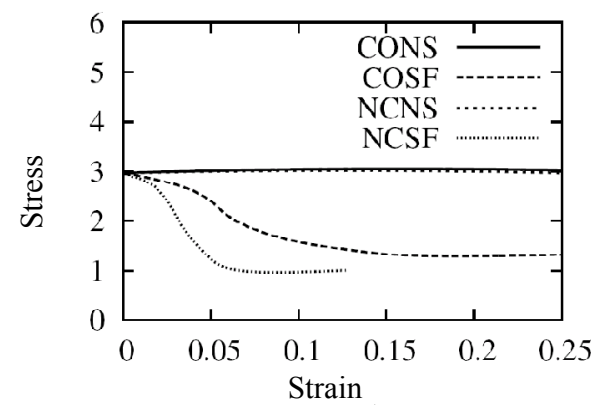

(b)

Fig. 3 Variation of average horizontal stress with horizontal strain in: (a) compression and (b) extension.

average normal stress on the right boundary with the horizontal strain, $\varepsilon$, for various material models. The $\mathrm{CO}$ (coaxial) model results from the non-coaxial angle $v=0$ and $\mathrm{NC}$ (non-coaxial) model has a non-coaxial angle $v=\phi$. The cohesion $c=c_{0}$ gives the NS (non-softening) case. The SF (softening) effect is governed by Eq. (14). The clear effects of non-coaxiality and the softening can be seen under compression. The non-coaxial material is weaker than the coaxial material (the stress is smaller). The softening effect starts at a very early stage and the stress drops in the initial softening phase. It is noted that the stress at the start of simulation is different for coaxial and non-coaxial flow rule. This is due to the choice of a high boundary velocity which makes the deformation plastic so that the non-coaxiality takes effect at start. This difference disappears when low value $(V=0.35)$ is used.

Fig. 4 shows the distribution of the second invariant of strain rate $\dot{\gamma}=\sqrt{D_{i j} D_{i j}}$ for deformed configurations of various material models under compression at strain level $\varepsilon=0.1$. Shear bands which emanate from the weaker zone in which the invariant of the strain rate concentrates are clearly seen in all four types of materials. For coaxial materials, the bands start relatively straight. When the material does not soften, they remain relatively straight. At later stages, these two bands disappear and instead one single band merges and runs from lower corner of the domain where the weak zone is no longer in the shear band. If the material softens, then the bands become concave curved (Fig. 4b) and at later stages multiple crossing bands merge. The central part of the top surface has concave shape (Figs. 4a and 4b). For non-coaxial materials, the bands start convex curved and then straighten gradually. Convex curved shear bands are also obtained by Lemiale et al. [23] for high friction. The central part of top surface has convex shape (Figs. $4 c$ and $4 d)$.

\subsection{Effects in Extension}

Fig. $3 \mathrm{~b}$ shows the variation of the average normal stress with the strain under extension for various material models. If there is no softening, then non-coaxiality has very little effect. For softening materials, non-coaxiality has a big effect and the stress drops in the initial softening stage. The stress increases again under increasing strain through the thinning of the layer thickness; the latter causing a decrease in the effect of gravity.

Fig. 5 shows the distribution of the second invariant of strain rate with deformed configuration for various models under extension at strain level $\varepsilon=0.1$. For coaxial material without softening, there are less distinguishable bands emerging from the weak zone where the second invariant of the strain rate in the bands does not differ much from that in the remaining region (Fig. 5a). The bands disappear at a later stage. If the material softens, then there is no clear picture with two bands. Instead multiple bands merge and develop and a strain concentrating region is found at the opposite site of the weak zone (Fig. 5b). At later stages, two bands merge from the region and join the neighboring bands. For non-coaxial materials, three 


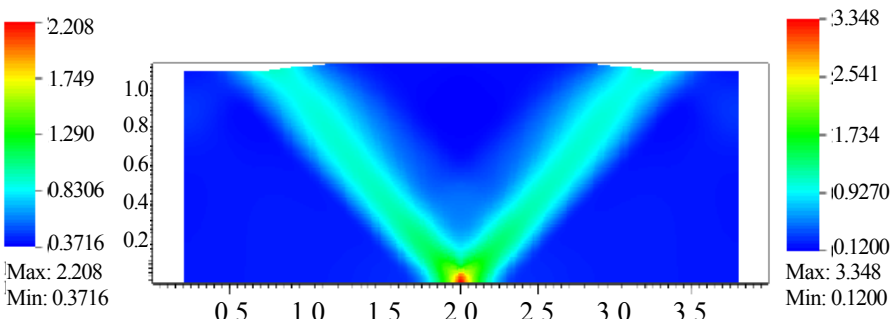

(a)
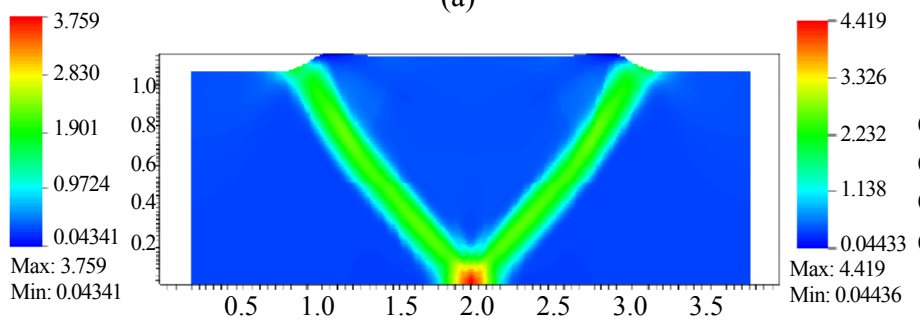

(c)

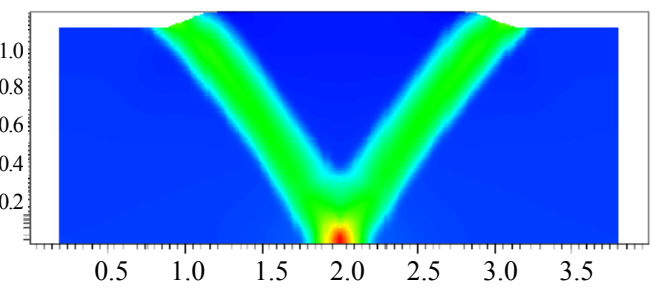

(b)

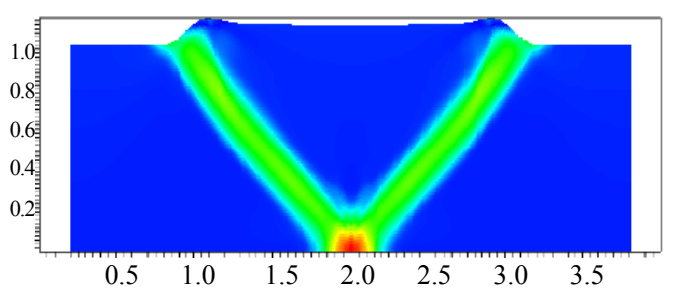

(d)

Fig. 4 Distribution of second invariant of strain rate under compression at strain $\varepsilon=0.1$ for four types of materials: (a) CO/NS, (b) CO/ SF, (c) NC/NS and (d) NC/SF.

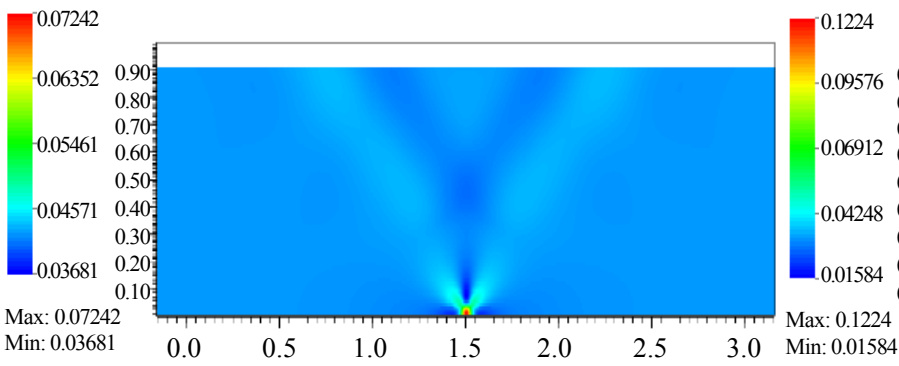

(a)

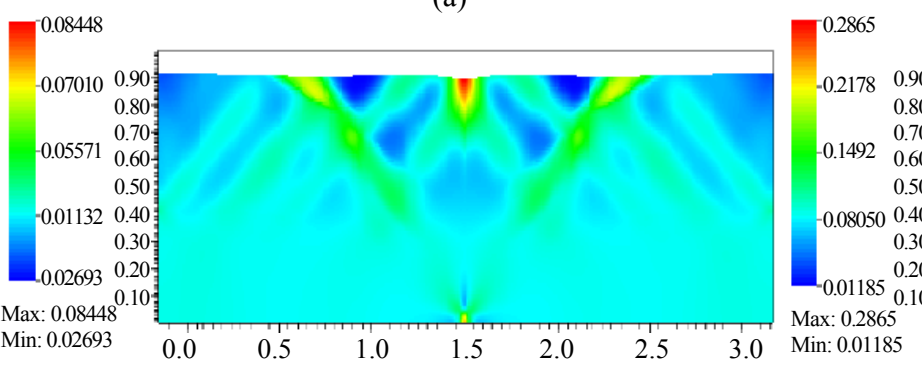

(c)

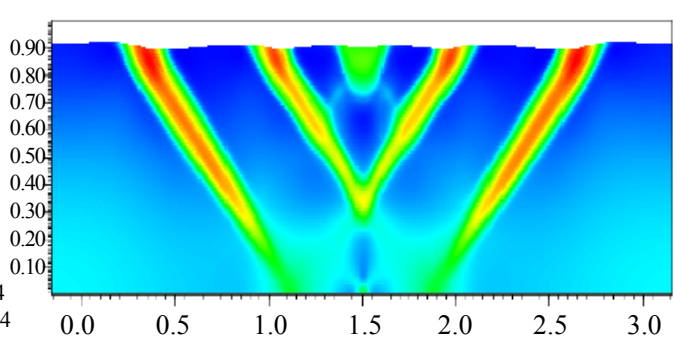

(b)

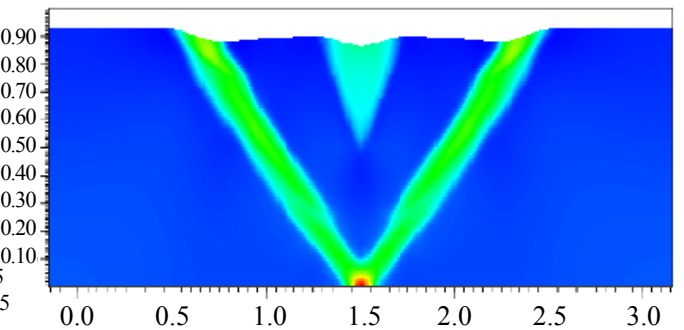

(d)

Fig. 5 Distribution of second invariant of strain rate under extension at strain $\varepsilon=0.1$ for four types of materials: (a) CO/NS, (b) $\mathrm{CO} / \mathrm{SF}$, (c) NC/NS and (d) NC/SF.

strain concentrating regions start on the top surface. The two off-centre surface concentrations correspond to outcrops of shear bands nucleated from the bottom centre perturbation and the centre surface concentration represents an outcrop of a tensile-separation (Mode I) fracture. If the material does not soften, extension of the bands terminates and more short bands merge from the top surface (Fig. 5c). If the material softens, then the bands from the two off-centre regions continue to extend and reach the weak zone (Fig. 5d). This pattern remains until end of the simulation.

\section{Conclusions}

In this paper, we formulate a simple viscous-plastic constitutive relation based on non-coaxial flow rule with single slip mechanism. Numerical simulations of rectangular domain with compression and extension 
conditions are carried out. Shear bands are formed in most cases. The non-coaxiality yields curved shear bands at the beginning in compression case. In extension case, multiple bands can form.

As deformation proceeds, the directions of principal stress and plastic strain rate change. Thus the angle of non-coaxiality varies with deformation. The angle of non-coaxiality is taken as a material parameter and is assumed to be constant in the numerical simulation in this paper. In a further study, we will calculate the angle of non-coaxiality.

\section{Acknowledgments}

The support by the ARC (Australian Research Council) through the discovery grants DP120102188: Hydraulic erosion of granular structures is gratefully acknowledged and DP140100490: qualitative and quantitative modelling of hydraulic fracturing of brittle materials.

\section{References}

[1] R. Hill, The Mathematical Theory of Plasticity, Oxford University Press, Oxford, 1998.

[2] J. Lubliner, Plasicity Theory, Macmillan Publishing, New York, 1990.

[3] A.B. Watts, J.H. Bodine, M.S. Steckler, Observation of flexure and the state of stress in the oceanic lithosphere, Journal Geophys Research 85 (1980) 6369-6376.

[4] M.A. Biot, The Mechanics of Incremental Deformations, Wiley, New York, 1965.

[5] W.R. Buck, Models of continental lithosphere extension, Journal Geophys Research 96 (B12) (1991) 20161-20178.

[6] P. England, Constraints on extension of continental lithosphere, Journal Geophys Research 88 (B2) (1983) 1145-1152.

[7] A.D. Gibbs, Structural evolution of extensional basin margin, Journal Geophys Research 141 (1984) 609-620.

[8] R. Hill, J.W. Hutchinson, Bifurcation phenomena in the plane strain tension test, Journal Mechanics Physics Solids 23 (1975) 239-264.

[9] L. Moresi, H.B. Mühlhaus, Anisotropic viscous models of large-deformation Mohr-Coulomb failure, Philosophical Magazine 86 (2006) 3287-3305.

[10] V. Tvergaard, A. Needleman, K.K. Lo, Flow localization in the plane strain tensile test, Journal Mechanics Physics Solids 29 (1981) 115-142.

[11] J. Walsh, J. Watterson, G. Yielding, The importance of small-scale faulting in regional extension, Nature 351 (1991) 391-393.

[12] W.F. Chen, Limit Analysis and Soil Plasticity, Elsevier, Amsterdam, 1975.

[13] J.W. Rudnicki, J.R. Rice, Conditions for the localization of deformation in pressure sensitive dilatant materials, Journal Mechanics Physics Solids 23 (1975) 337-351.

[14] I. Vardoulakis, J. Sulem, Bifurcation Analysis in Geomechanics, Chapman and Hall, London, 1995.

[15] G. de Josselin de Jong, The undefiniteness in kinematics for friction materials, in: Proceedings of the Conference Earth Pressure Problem, Brussels, 1958, pp. 55-70.

[16] G. de Josselin de Jong, The double sliding, free rotating model for granular assemblies, Geotechnique 21 (3) (1971) 155-162.

[17] A.J.M. Spencer, A theory of the kinematics of ideal soils under plane strain conditions, Journal Mechanics Physics Solids 12 (1964) 337-351.

[18] A.J.M. Spencer, Deformation of Ideal Granular Materials, Mechanics of Solids: The Rodney Hill Anniversary Volume, Pergamon Press, Oxford, 1982, pp. 607-652.

[19] M.M. Mehrabadi, S.C. Cowin, On the double sliding free rotating model for the deformation of granular materials, Journal Mechanics Physics Solids 29 (1981) 269-282.

[20] M.J. Jiang, D. Harris, H.S. Yu, Kinematic models for non-coaxial granular materials, Part I, International Journal Numerical Analytical Methods Geomechanics 29 (2005) 643-661.

[21] M.J. Jiang, D. Harris, H.S. Yu, Kinematic models for non-coaxial granular materials, Part II, International Journal Numerical Analytical Methods Geomechanics 29 (2005) 663-689.

[22] A. Tordesillas, J. Shi, H.B. Muhlhaus, Non-coaxiality and force chain evolution, International Journal Engineering Science 47 (2009) 1386-1404.

[23] V. Lemiale, H. Muhlhaus, L. Moresi, J. Stafford, Shear banding analysis of plastic models formulated for incompressible viscous flows, Physics Earth and Planetary Interiors 171 (2008) 177-186.

[24] L. Anand, Plane deformations of ideal granular materials, Journal Mechanics Physics Solids 31 (1983) 105-122.

[25] D. Harris, A unified formulation for plasticity models of granular and other materials, Proceedings of Royal Society London A 450 (1995) 37-49.

[26] H.B. Muhlhaus, L. Moresi, L. Gross, J. Grotowski, The influence of non-coaxiality on shear banding in viscous-plastic materials, Granular Matter 12 (3) (2010) 229-238.

[27] O.C. Zienkiewicz, R.L. Taylor, The Finite Element Method, 4th ed., McGraw Hill Book Company, London, 1994.

[28] H.L. Schreyer, Analytical solutions for nonlinear 
strain-gradient softening and localization, Journal Applied Mechanics 57 (1990) 522-529.

[29] A. Needleman, Material rate dependence and mesh sensitivity in localization problems, Computer Methods
Applied Mechanics Engineering 67 (1988) 69-85.

[30] H.B. Mühlhaus, I. Vardoulakis, The thickness of shear bands in granular materials, Geotechnique 37 (1987) 271-283. 